\title{
Excited States of Crystalline Point Defects with Multireference Density Matrix Embedding
}

\section{Theory}

Abhishek Mitra, ${ }^{\dagger, \|}$ Hung Q. Pham, ${ }^{\ddagger}, \|$ Riddhish Pandharkar, ${ }^{\dagger}$ Matthew R.

$$
\text { Hermes, }{ }^{\dagger} \text { and Laura Gagliardi*, }, \uparrow
$$

$\dagger$ Department of Chemistry, Chicago Center for Theoretical Chemistry, University of Chicago, Chicago, IL 60637, USA.

$\ddagger$ Department of Chemistry,Smith Hall, 207 Pleasant St SE, Minneapolis, MN 55455-0431, $U S A$

9Argonne National Laboratory 9700 S. Cass Avenue Lemont, IL 60439, USA.

$\S$ Department of Chemistry, Pritzker School of Molecular Engineering, James Franck Institute, Chicago Center for Theoretical Chemistry, University of Chicago, Chicago, IL 60637, USA.

\|Contributed equally to this work

E-mail: Igagliardi@uchicago.edu

\section{Contents}

S01 Computational details $\ldots \ldots \ldots \ldots \ldots \ldots \ldots$

S02 $a_{2 u}$-like orbital in the ROHF bath $\ldots \ldots \ldots \ldots \ldots$

S03 Excitation energies for OV on a $\mathrm{MgO}(100)$ surface using a 2,2 active space $\ldots \quad$. 7 
S04 Total energies for supercells considered for OV in $\mathrm{Mg}(100)$ surface . . . . . . . . 8

S05 Convergence of excitation energies in plane . . . . . . . . . . . . . . . 11

S06 Excitation energies using the $\mathrm{SiC}_{6}$ impurity cluster in the silicon vacancy defect $\mathbf{1 3}$

S07 Active spaces explored for the silicon vacancy defect . . . . . . . . . . . . . 14

S08 Total energies for the cluster calculations for the silicon vacancy defect . . . . 15

S09 Total Energies for supercells considered in the silicon vacancy defect . . . . . 16

S010 Active spaces explored within this work . . . . . . . . . . . . . 17

S011 Natural occupation numbers. . . . . . . . . . . . . . . . . . . . 20

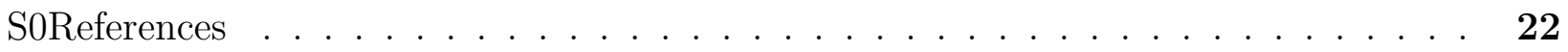




\section{S01. Computational details}

All DMET calculations are performed using our in-house pDMET code which utilizes the electron integrals and quantum chemical solvers from the PySCF package. ${ }^{1-3}$ Wannier90 ${ }^{4}$ is used to construct MLWFs via the pyWannier90 interface. ${ }^{3}$ In the Wannierisation procedure, all available bands are used for the calculations. Electron integrals and $\Gamma$-point (non-embedding) calculations are obtained by means of PySCF. We also employ the efficient algorithm for the orbital transformation to the embedding space which has been introduced recently by Cui et al. ${ }^{5}$ The spin-restricted formalism is used in all the calculations. The GTH pseudopotentials ${ }^{6}$ is used for all the calculations.

The geometry optimizations are performed at the spin-unrestricted PBEsol level ${ }^{7}$ using the Vienna Ab initio Simulation Package (VASP). ${ }^{8-11}$ The convergence criteria of $10^{-6} \mathrm{eV}$ and $10^{-3} \mathrm{eV} / \AA$ are used for the energy and force, respectively.

Oxygen vacancy model (OV): First, we consider a single layer model, denoted as $\mathrm{Mg}_{18} \mathrm{O}_{18}$, to represent the $\mathrm{MgO}(100)$ surface. The oxygen vacancy defects are created by removing the neutral oxygen atom at the center of the unit cell. We use a vacuum of 23.518 $\AA$ to separate the layer and its periodic images along the [100] direction. In the DMET calculations, we place a dummy oxygen atom at the vacancy to provide basis functions to span the electron density of the defect. The polarized triple-zeta basis set TZVP is used for the dummy oxygen and eight nearest atoms (4 $\mathrm{O}$ and $4 \mathrm{Mg}$ ) while the polarized double-zeta basis set DZVP is used for all other atoms. The first singlet-singlet excitation is computed via the state-averaging formalism of $\mathrm{CASSCF}^{12-14}$ with equal weights for the first two roots. The singlet-triplet excitation is computed using the difference between a state-specific CASSCF for the triplet state and the first root of the state-averaged singlet calculations. The double and triple layered model are constructed by placing non-defective one and two layers of $\mathrm{Mg}_{18} \mathrm{O}_{18}$ below the first layer respectively. For these models the polarized triple-zeta basis set TZVP is used for the dummy oxygen and nine nearest atoms (4 $\mathrm{O}$ and $5 \mathrm{Mg}$ ) while the 
polarized double-zeta basis set DZVP is used for all other atoms. The orbitals in the $(2,8)$ active space and for the two and three layered systems do not transform as irreps therefore we use $S_{n}$ to label the $n$th excited singlet states and $T_{n}$ to label $n$th triplet states.

Neutral silicon vacancy in carbon diamond $\left(\mathrm{SiV}^{0}\right)$ : The silicon vacancy defect is created by removing two carbons followed by inserting a silicon atom in the midpoint. A $2 \times 2 \times 2$ supercell of the primitive cell of the carbon diamond crystal is used for numerical testing, resulting in a $\mathrm{SiC}_{52}$ model with 53 atoms. For extension to realistic models a $4 \times 4 \times 4$ supercell of the primitive cell of the carbon diamond crystal and a $3 \times 3 \times 3$ supercell of the 8-atom unit cell of the carbon diamond crystal are used resulting in $\mathrm{SiC}_{126}$ model with 127 atoms and $\mathrm{SiC}_{214}$ model with 215 atoms respectively. We use $S_{n}$ and $T_{n}$ for labelling the $n$th excited singlet and triplet states respectively. We use the polarized triple-zeta basis set TZVP for Si, the polarized double-zeta basis set DZVP for the six nearest carbons with respect to the Si site, and the double-zeta basis set DZV for the rest. ${ }^{15} \mathrm{We}$ use the stateaverage CASSCF formalism by averaging over 4 singlet states with equal weights to obtain singlet state energies to and 6 triplet states with equal weights to obtain triplet state energies. An active space of 10 electrons in 12 orbitals was used and has been denoted as $(10,12)$ For computing all excitation energies, the triplet ground state is considered as a reference.

Cluster calculations for the silicon vacancy in carbon diamond $\left(\mathrm{SiV}^{0}\right)$ : A cluster of the Si vacancy -consisting of the silicon and 54 carbons (3 coordination spheres around the $\mathrm{Si}$ ) - was cut from the optimized periodic structure. The dangling bonds of the carbons on the edges were capped with an appropriate number of hydrogen atoms to maintain the neutrality of the cluster. The positions of the hydrogens were optimized with all other atom positions frozen using M06-L/def2-svp level of theory in Gaussian16. The CASSCF and NEVPT2 calculation on the cluster were performed in Orca (version 4.2.0) with cc-pvdz basis on the carbons and the cc-pvtz basis on the silicon atom. Density fitting with resolution of identity and numerical chain-of-sphere integration (RIJCOSX) was used to reduce the cost of computation. The strongly contracted (SC) NEVPT2 was used in all cases. Separate state 
average calculations for the singlet and triplet multiplicities with 4 and 6 states respectively were performed with an active space of 10 electrons in 12 orbitals $(10,12)$.

Identification of the defect states: First, the molecular orbitals $\mathbf{C}$ (equivalently, the Bloch orbitals in a $\Gamma$-point sampling calculation) are orthogonalized using the overlap matrix S:

$$
\tilde{\mathbf{C}}=\mathbf{S}^{1 / 2} \mathbf{C}
$$

For each orbital, the weight $\left(\mathrm{w}_{i}\right)$ of the defect site $\mathbf{X}$ is calculated by:

$$
\mathrm{w}_{i}=100 \times \frac{\sum_{\mu \subset \mathbf{X}} \tilde{\mathrm{C}}_{\mu i}^{2}}{\sum_{\mu} \tilde{\mathrm{C}}_{\mu i}^{2}}
$$

Defect states are the orbitals that are in close proximity to Fermi energy and exhibit a significant contribution from $\mathbf{X}$. For OV, $\mathbf{X}$ is the dummy atom, and the two defect states $a_{1 g}$ and $a_{2 u}$ exhibit a weight of 17.4 and $18.6 \%$, respectively. Similarly, $\mathbf{X}$ is the silicon atom for the $\mathrm{SiV}^{0}$ system, and the four defect states $e_{u x}, e_{u y}, e_{g x}$ and $e_{g y}$ exhibit a weight of 13.4, $13.4,20.6$, and $20.6 \%$, respectively .

\section{S02. $a_{2 u}$-like orbital in the ROHF bath}

We show here an example of the $a_{2 u}$-like orbital in the embedding space generated by the $\mathrm{ROHF}$ bath when the $\mathrm{OV}+\mathrm{Mg}_{4}$ impurity cluster is used which is absent from the RHF baths. 
[100]

ㄷ

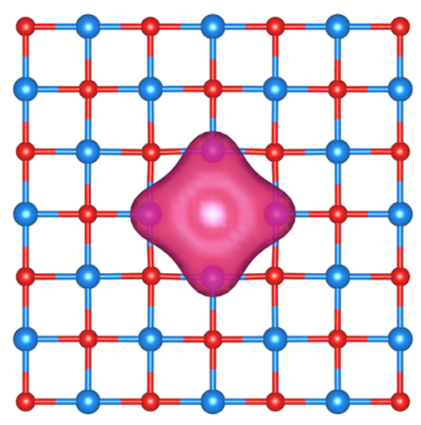

[001]

(อ)

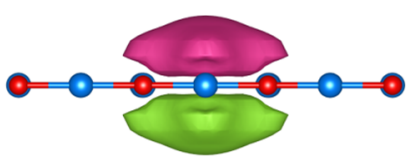

Figure 1: The $a_{2 u}$-like orbital in the embedding space generated by the ROHF bath when the $\mathrm{OV}+\mathrm{Mg}_{4}$ impurity cluster is used. A similar orbital is absent when the RHF bath is employed. 


\section{S03. Excitation energies for $\mathrm{OV}$ on a $\mathrm{MgO}(100)$ surface using a 2,2 active space}

Table 1: Vertical excitation energies (in eV) of the oxygen vacancy on the $\mathrm{MgO}(100)$ surface obtained using DMET with CASSCF and NEVPT2 using an active space of 2 electrons in 2 orbitals. The extrapolated CAS-DMET and NEVPT2-DMET energies from the linear regression are labeled as "Extrap". The excitation energies preceeded by a star correspond to experimental numbers.

\begin{tabular}{|c|c|c|c|c|c|}
\hline Excitation & Layers & Impurity cluster & CASSCF & NEVPT2 & Literature \\
\hline$S_{0} \rightarrow T_{1}$ & $\begin{array}{l}\mathrm{Mg}_{36} \mathrm{O}_{36} \\
\mathrm{Mg}_{54} \mathrm{O}_{54}\end{array}$ & $\begin{array}{l}\mathrm{OV}+\mathrm{Mg}_{4} \\
\mathrm{OV}+\mathrm{Mg}_{4} \mathrm{O}_{4} \\
\mathrm{OV}+\mathrm{Mg}_{4} \mathrm{O}_{8} \\
\text { Extrap } \\
\mathrm{OV}+\mathrm{Mg}_{4} \\
\mathrm{OV}+\mathrm{Mg}_{4} \mathrm{O}_{4} \\
\mathrm{OV}+\mathrm{Mg}_{4} \mathrm{O}_{5} \\
\text { Extrap } \\
\mathrm{OV}+\mathrm{Mg}_{4} \\
\mathrm{OV}+\mathrm{Mg}_{4} \mathrm{O}_{4} \\
\mathrm{OV}+\mathrm{Mg}_{4} \mathrm{O}_{5} \\
\text { Extrap }\end{array}$ & $\begin{array}{l}1.30 \\
1.32 \\
1.32 \\
1.33 \\
1.67 \\
1.70 \\
1.69 \\
1.71 \\
1.61 \\
1.64 \\
1.63 \\
1.65\end{array}$ & $\begin{array}{l}1.91 \\
2.09 \\
2.12 \\
2.18 \\
2.10 \\
2.25 \\
2.27 \\
2.42 \\
2.04 \\
2.19 \\
2.20 \\
2.36\end{array}$ & $1.93^{16}$ \\
\hline$S_{0} \rightarrow S_{1}$ & $\begin{array}{l}\mathrm{Mg}_{18} \mathrm{O}_{18} \\
\mathrm{Mg}_{36} \mathrm{O}_{36} \\
\mathrm{Mg}_{54} \mathrm{O}_{54}\end{array}$ & $\begin{array}{l}\mathrm{OV}+\mathrm{Mg}_{4} \\
\mathrm{OV}+\mathrm{Mg}_{4} \mathrm{O}_{4} \\
\mathrm{OV}+\mathrm{Mg}_{4} \mathrm{O}_{8} \\
\text { Extrap } \\
\mathrm{OV}+\mathrm{Mg}_{4} \\
\mathrm{OV}+\mathrm{Mg}_{4} \mathrm{O}_{4} \\
\mathrm{OV}+\mathrm{Mg}_{4} \mathrm{O}_{5} \\
\text { Extrap } \\
\mathrm{OV}+\mathrm{Mg}_{4} \\
\mathrm{OV}+\mathrm{Mg}_{4} \mathrm{O}_{4} \\
\mathrm{OV}+\mathrm{Mg}_{4} \mathrm{O}_{5} \\
\text { Extrap }\end{array}$ & $\begin{array}{l}3.27 \\
3.26 \\
3.25 \\
3.25 \\
3.81 \\
3.73 \\
3.67 \\
3.59 \\
3.70 \\
3.64 \\
3.57 \\
3.50\end{array}$ & $\begin{array}{l}3.17 \\
3.05 \\
3.00 \\
2.97 \\
3.80 \\
3.73 \\
3.60 \\
3.53 \\
3.68 \\
3.63 \\
3.53 \\
3.46\end{array}$ & $\begin{array}{c}3.24^{16} \\
* 2.30^{17} \\
* 1.0, * 1.3, * 2.4, * 3.4^{18} \\
* 1.2, * 3.6, * 5.3^{19}\end{array}$ \\
\hline
\end{tabular}

In Table 2 of the main text, we report all excitation energies using the $(2,8)$ active space. Here we report the excitation energies using the smaller $(2,2)$ active space. We observe the same trend as for the higher active space. A thing of note is that the CASSCF method recovers more dynamic correlation in a larger active space and is therefore closer to the 
NEVPT2 excitation energies when an active space of $(2,8)$ is used.

S04. Total energies for supercells considered for $\mathrm{OV}$ in $\mathrm{Mg}(100)$ surface

Total energies for the $\mathrm{OV}+\mathrm{Mg}_{4}$ impurity cluster

Table 2: Total energy per unit cell (in Hartree) at the SA-CASSCF and NEVPT2 level for $\mathrm{Mg}_{18} \mathrm{O}_{18}$ (single layer)

\begin{tabular}{cccccc}
\hline \multicolumn{5}{c}{ DMET } & \multicolumn{2}{c}{ Full } \\
\hline $\begin{array}{c}\text { Mean- } \\
\text { field }\end{array}$ & State & SA-CASSCF & NEVPT2 & SA-CASSCF & NEVPT2 \\
\hline RHF & ${ }^{1} A_{1 g}$ & -1390.27568 & -1390.46596 & -1390.27047 & -612.45635 \\
& ${ }^{1} A_{2 u}$ & -1390.07804 & -1390.27825 & -1390.15093 & -612.31920 \\
& ${ }^{3} A_{2 u}$ & -1390.17654 & -1390.34767 & -1390.22152 & -612.35895 \\
ROHF & ${ }^{1} A_{1 g}$ & -1390.26945 & -1390.47172 & -1390.27047 & -612.45635 \\
& ${ }^{1} A_{2 u}$ & -1390.14933 & -1390.35535 & -1390.15093 & -612.31920 \\
& ${ }^{3} A_{2 u}$ & -1390.22152 & -1390.40138 & -1390.22152 & -612.35895 \\
\hline
\end{tabular}

Table 3: Total energy per unit cell (in Hartree) at the SA-CASSCF and NEVPT2 level for $\mathrm{Mg}_{36} \mathrm{O}_{36}$ (two layers)

\begin{tabular}{cccccc}
\hline & & \multicolumn{2}{c}{ DMET $(2,2)$} & \multicolumn{2}{c}{ DMET $(2,8)$} \\
\hline $\begin{array}{c}\text { Mean- } \\
\text { field }\end{array}$ & State & SA-CASSCF & NEVPT2 & SA-CASSCF & NEVPT2 \\
\hline ROHF & $\mathrm{S}_{0}$ & -2795.54708 & -2795.73185 & -2795.56846 & -2795.73368 \\
& $\mathrm{~S}_{1}$ & -2795.40724 & -2795.59231 & -2795.42120 & -2795.59028 \\
& $\mathrm{~T}_{1}$ & -2795.48559 & -2795.65456 & -2795.48792 & -2795.65323 \\
\hline
\end{tabular}

Table 4: Total energy per unit cell (in Hartree) at the SA-CASSCF and NEVPT2 level for $\mathrm{Mg}_{54} \mathrm{O}_{54}$ (three layers)

\begin{tabular}{cccccc}
\hline & \multicolumn{2}{c}{ DMET $(2,2)$} & \multicolumn{2}{c}{ DMET $(2,8)$} \\
\hline $\begin{array}{c}\text { Mean- } \\
\text { field }\end{array}$ & State & SA-CASSCF & NEVPT2 & SA-CASSCF & NEVPT2 \\
\hline ROHF & $\mathrm{S}_{0}$ & -4200.86556 & -4201.04409 & -4200.88719 & -4201.04623 \\
& $\mathrm{~S}_{1}$ & -4200.72972 & -4200.90897 & -4200.74385 & -4200.90687 \\
& $\mathrm{~T}_{1}$ & -4200.80628 & -4200.96902 & -4200.80855 & -4200.96784 \\
\hline
\end{tabular}




\section{Total energies for the $\mathrm{OV}+\mathrm{Mg}_{4} \mathrm{O}_{4}$ impurity cluster}

Table 5: Total energy per unit cell (in Hartree) at the SA-CASSCF and NEVPT2 level for $\mathrm{Mg}_{18} \mathrm{O}_{18}$ (single layer)

\begin{tabular}{cccccc}
\hline & \multicolumn{2}{c}{ DMET } & \multicolumn{2}{c}{ Full } \\
\hline $\begin{array}{c}\text { Mean- } \\
\text { field }\end{array}$ & State & SA-CASSCF & NEVPT2 & SA-CASSCF & NEVPT2 \\
\hline RHF & ${ }^{1} A_{1 g}$ & -1390.27250 & -1391.32188 & -1390.27047 & -612.45635 \\
& ${ }^{1} A_{2 u}$ & -1390.26991 & -1391.32493 & -1390.27047 & -612.31920 \\
& ${ }^{3} A_{2 u}$ & -1390.20695 & -1391.22879 & -1390.22152 & -612.45309 \\
ROHF & ${ }^{1} A_{1 g}$ & -1390.12690 & -1391.18678 & -1390.15093 & -612.45635 \\
& ${ }^{1} A_{2 u}$ & -1390.15025 & -1391.21270 & -1390.15093 & -612.31920 \\
& ${ }^{3} A_{2 u}$ & -1390.22152 & -1391.24796 & -1390.22152 & -612.35895 \\
\hline
\end{tabular}

Table 6: Total energy per unit cell (in Hartree) at the SA-CASSCF and NEVPT2 level for $\mathrm{Mg}_{36} \mathrm{O}_{36}$ (two layers)

\begin{tabular}{cccccc}
\hline & \multicolumn{2}{c}{ DMET $(2,2)$} & \multicolumn{2}{c}{ DMET $(2,8)$} \\
\hline $\begin{array}{c}\text { Mean- } \\
\text { field }\end{array}$ & State & SA-CASSCF & NEVPT2 & SA-CASSCF & NEVPT2 \\
\hline ROHF & $\mathrm{S}_{0}$ & -2795.54786 & -2796.58592 & -2795.57063 & -2796.57996 \\
& $\mathrm{~S}_{1}$ & -2795.41087 & -2796.44881 & -2795.42481 & -2796.43824 \\
& $\mathrm{~T}_{1}$ & -2795.48556 & -2796.50307 & -2795.48810 & -2796.49714 \\
\hline
\end{tabular}

Table 7: Total energy per unit cell (in Hartree) at the SA-CASSCF and NEVPT2 level for $\mathrm{Mg}_{54} \mathrm{O}_{54}$ (three layers)

\begin{tabular}{cccccc}
\hline & \multicolumn{2}{c}{ DMET $(2,2)$} & \multicolumn{2}{c}{ DMET $(2,8)$} \\
\hline $\begin{array}{c}\text { Mean- } \\
\text { field }\end{array}$ & State & SA-CASSCF & NEVPT2 & SA-CASSCF & NEVPT2 \\
\hline ROHF & $\mathrm{S}_{0}$ & -4200.86640 & -4201.90574 & -4200.88922 & -4201.90069 \\
& $\mathrm{~S}_{1}$ & -4200.73268 & -4201.77248 & -4200.74698 & -4201.76277 \\
& $\mathrm{~T}_{1}$ & -4200.80625 & -4201.82522 & -4200.80874 & -4201.82017 \\
\hline
\end{tabular}

Total energies for the $\mathrm{OV}+\mathrm{Mg}_{5} \mathrm{O}_{4}$ impurity cluster 
Table 8: Total energy per unit cell (in Hartree) at the SA-CASSCF and NEVPT2 level for $\mathrm{Mg}_{36} \mathrm{O}_{36}$ (two layers)

\begin{tabular}{cccccc}
\hline \multicolumn{1}{c}{ DMET $(2,2)$} & \multicolumn{2}{c}{ DMET $(2,8)$} \\
\hline $\begin{array}{c}\text { Mean- } \\
\text { field }\end{array}$ & State & SA-CASSCF & NEVPT2 & SA-CASSCF & NEVPT2 \\
\hline ROHF & $\mathrm{S}_{0}$ & -2795.54761 & -2796.66435 & -2795.57166 & -2796.65850 \\
& $\mathrm{~S}_{1}$ & -2795.41278 & -2796.53187 & -2795.42795 & -2796.52063 \\
& $\mathrm{~T}_{1}$ & -2795.48557 & -2796.58079 & -2795.48845 & -2796.57457 \\
\hline
\end{tabular}

Table 9: Total energy per unit cell (in Hartree) at the SA-CASSCF and NEVPT2 level for $\mathrm{Mg}_{54} \mathrm{O}_{54}$ (three layers)

\begin{tabular}{cccccc}
\hline & & \multicolumn{2}{c}{ DMET $(2,2)$} & \multicolumn{2}{c}{ DMET $(2,8)$} \\
\hline $\begin{array}{c}\text { Mean- } \\
\text { field }\end{array}$ & State & SA-CASSCF & NEVPT2 & SA-CASSCF & NEVPT2 \\
\hline ROHF & $\mathrm{S}_{0}$ & -4200.86618 & -4201.96228 & -4200.89015 & -4201.95762 \\
& $\mathrm{~S}_{1}$ & -4200.73499 & -4201.83272 & -4200.75014 & -4201.82266 \\
& $\mathrm{~T}_{1}$ & -4200.80627 & -4201.88132 & -4200.80906 & -4201.87623 \\
\hline
\end{tabular}

Total energies for the $\mathrm{OV}+\mathrm{Mg}_{4} \mathrm{O}_{8}$ impurity cluster

Table 10: Total energy per unit cell (in Hartree) at the SA-CASSCF and NEVPT2 level for $\mathrm{Mg}_{18} \mathrm{O}_{18}$ (single layer)

\begin{tabular}{cccccc}
\hline \multicolumn{1}{c}{ DMET } & \multicolumn{2}{c}{ Full } \\
\hline $\begin{array}{c}\text { Mean- } \\
\text { field }\end{array}$ & State & SA-CASSCF & NEVPT2 & SA-CASSCF & NEVPT2 \\
\hline RHF & ${ }^{1} A_{1 g}$ & -1390.27061 & -1392.33765 & -1390.27047 & -612.45635 \\
& ${ }^{1} A_{2 u}$ & -1390.27011 & -1392.43669 & -1390.27047 & -612.31920 \\
& ${ }^{3} A_{2 u}$ & -1390.22025 & -1392.25747 & -1390.22152 & -612.35895 \\
ROHF & ${ }^{1} A_{1 g}$ & -1390.14947 & -1392.22561 & -1390.15093 & -612.45635 \\
& ${ }^{1} A_{2 u}$ & -1390.15067 & -1392.32638 & -1390.15093 & -612.31920 \\
& ${ }^{3} A_{2 u}$ & -1390.22152 & -1392.35864 & -1390.22152 & -612.35895 \\
\hline
\end{tabular}




\section{S05. Convergence of excitation energies in plane}

Having accounted for dependence with the number of layers in the main text, we check here for the dependence by extension in the other two dimensions, i.e. in plane (Figure 2) using both $(2,2)$ and $(2,8)$ active spaces. We find in Tables 1112 that all excitation energies differ by less than $0.1 \mathrm{eV}$. Therefore the defect-defect interactions of $\mathrm{Mg}_{18} \mathrm{O}_{18}$ between periodic images can be said to be minimal.
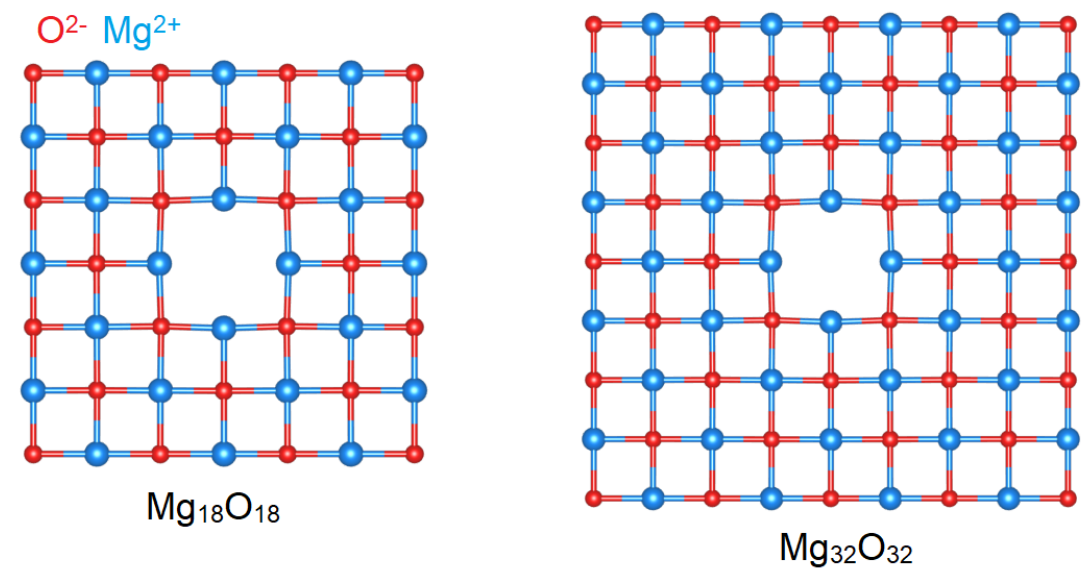

Figure 2: The $\mathrm{Mg}_{18} \mathrm{O}_{18}$ (left) single layer and the extended $\mathrm{Mg}_{32} \mathrm{O}_{32}$ (right) single layer considered to check for in-plane convergence of excitation energies. 
Table 11: Vertical excitation energies (in eV) of the oxygen vacancy on the $\mathrm{MgO}(100)$ surface between $\mathrm{Mg}_{18} \mathrm{O}_{18}$ and $\mathrm{Mg}_{32} \mathrm{O}_{32}$ supercells obtained using DMET with CASSCF and NEVPT2 using an active space of 2 electrons in 2 orbitals. The extrapolated CAS-DMET and NEVPT2-DMET energies from the linear regression are labeled as "Extrap". The excitation energies preceeded by a star correspond to experimental numbers.

\begin{tabular}{cclcc}
\hline Excitation & Layers & Impurity cluster & CASSCF & NEVPT2 \\
\hline$S_{0} \rightarrow T_{1}$ & $\mathrm{Mg}_{18} \mathrm{O}_{18}$ & $\mathrm{OV}+\mathrm{Mg}_{4}$ & 1.30 & 1.91 \\
& & $\mathrm{OV}+\mathrm{Mg}_{4} \mathrm{O}_{4}$ & 1.32 & 2.09 \\
& & $\mathrm{OV}+\mathrm{Mg}_{4} \mathrm{O}_{8}$ & 1.32 & 2.12 \\
& & Extrap & 1.33 & 2.18 \\
& $\mathrm{Mg}_{32} \mathrm{O}_{32}$ & $\mathrm{OV}+\mathrm{Mg}_{4}$ & 1.29 & 1.89 \\
& & $\mathrm{OV}+\mathrm{Mg}_{4} \mathrm{O}_{4}$ & 1.30 & 2.07 \\
& & $\mathrm{OV}+\mathrm{Mg}_{4} \mathrm{O}_{8}$ & 1.31 & 2.09 \\
& & Extrap & 1.31 & 2.15 \\
\hline \multirow{2}{*}{$S_{0} \rightarrow S_{1}$} & $\mathrm{Mg}_{18} \mathrm{O}_{18}$ & $\mathrm{OV}+\mathrm{Mg}_{4}$ & 3.27 & 3.17 \\
& & $\mathrm{OV}+\mathrm{Mg}_{4} \mathrm{O}_{4}$ & 3.26 & 3.05 \\
& & $\mathrm{OV}+\mathrm{Mg}_{4} \mathrm{O}_{8}$ & 3.25 & 3.00 \\
& & Extrap & 3.25 & 2.97 \\
& $\mathrm{Mg}_{32} \mathrm{O}_{32}$ & $\mathrm{OV}+\mathrm{Mg}_{4}$ & 3.22 & 3.12 \\
& & $\mathrm{OV}+\mathrm{Mg}_{4} \mathrm{O}_{4}$ & 3.20 & 3.02 \\
& & $\mathrm{OV}+\mathrm{Mg}_{4} \mathrm{O}_{8}$ & 3.19 & 2.98 \\
& & Extrap & 3.19 & 2.95 \\
\hline
\end{tabular}


Table 12: A comparison of vertical excitation energies (in eV) of the oxygen vacancy on the $\mathrm{MgO}(100)$ surface between $\mathrm{Mg}_{18} \mathrm{O}_{18}$ and $\mathrm{Mg}_{32} \mathrm{O}_{32}$ supercells obtained using DMET with CASSCF and NEVPT2 using an active space of 2 electrons in 8 orbitals. The extrapolated CAS-DMET and NEVPT2-DMET energies from the linear regression are labeled as "Extrap". The excitation energies preceeded by a star correspond to experimental numbers.

\begin{tabular}{cclcc}
\hline Excitation & Layers & Impurity cluster & CASSCF & NEVPT2 \\
\hline$S_{0} \rightarrow T_{1}$ & $\mathrm{Mg}_{18} \mathrm{O}_{18}$ & $\mathrm{OV}+\mathrm{Mg}_{4}$ & 1.93 & 1.98 \\
& & $\mathrm{OV}+\mathrm{Mg}_{4} \mathrm{O}_{4}$ & 1.97 & 2.07 \\
& & $\mathrm{OV}+\mathrm{Mg}_{4} \mathrm{O}_{8}$ & 1.98 & 2.13 \\
& & Extrap & 1.99 & 2.11 \\
& $\mathrm{Mg}_{32} \mathrm{O}_{32}$ & $\mathrm{OV}+\mathrm{Mg}_{4}$ & 1.92 & 1.96 \\
& & $\mathrm{OV}+\mathrm{Mg}_{4} \mathrm{O}_{4}$ & 1.96 & 2.04 \\
& & $\mathrm{OV}+\mathrm{Mg}_{4} \mathrm{O}_{8}$ & 1.96 & 2.05 \\
& & Extrap & 1.97 & 2.08 \\
\hline \multirow{2}{*}{$S_{0} \rightarrow S_{1}$} & $\mathrm{Mg}_{18} \mathrm{O}_{18}$ & $\mathrm{OV}+\mathrm{Mg}_{4}$ & 3.48 & 3.37 \\
& & $\mathrm{OV}+\mathrm{Mg}_{4} \mathrm{O}_{4}$ & 3.46 & 3.34 \\
& & $\mathrm{OV}+\mathrm{Mg}_{4} \mathrm{O}_{8}$ & 3.45 & 3.30 \\
& & Extrap & 3.45 & 3.29 \\
& $\mathrm{Mg}_{32} \mathrm{O}_{32}$ & $\mathrm{OV}+\mathrm{Mg}_{4}$ & 3.43 & 3.32 \\
& & $\mathrm{OV}+\mathrm{Mg}_{4} \mathrm{O}_{4}$ & 3.41 & 3.29 \\
& & $\mathrm{OV}+\mathrm{Mg}_{4} \mathrm{O}_{8}$ & 3.40 & 3.26 \\
& & Extrap & 3.40 & 3.26 \\
\hline
\end{tabular}

S06. Excitation energies using the $\mathrm{SiC}_{6}$ impurity cluster in the silicon vacancy defect

Here, we use the smaller impurity cluster $\mathrm{SiC}_{6}$ to compute excitation energies for the models described in the main text. The convergence pattern is similar to that observed for the values obtained using the $\mathrm{SiC}_{12}$ impurity cluster. 
Table 13: Singlet and triplet excitation energies (in eV) of $\mathrm{SiV}^{0}$ computed using CASDMET and NEVPT2-DMET using the $\mathrm{SiC}_{6}$ impurity cluster shown in Figure $2 \mathrm{~b}$ of the main text. The Table shows excitation energies for the $\mathrm{SiC}_{52}, \mathrm{SiC}_{126}$ and $\mathrm{SiC}_{214}$ unit cells. All calculations use an active space of $(10,12)$. Computational values from literature are also included.

\begin{tabular}{ccccccccc}
\hline \multirow{2}{*}{ State } & \multicolumn{3}{c}{ CAS-DMET } & \multicolumn{3}{c}{ NEVPT2-DMET } & \multicolumn{2}{c}{ Literature } \\
\cline { 2 - 9 } & $\mathrm{SiC}_{52}$ & $\mathrm{SiC}_{126}$ & $\mathrm{SiC}_{214}$ & $\mathrm{SiC}_{52}$ & $\mathrm{SiC}_{126}$ & $\mathrm{SiC}_{214}$ & Comput. & Expt. \\
\hline $\mathrm{T}_{0}$ & 0.00 & 0.00 & 0.00 & 0.00 & 0.00 & 0.00 & & \\
$\mathrm{~T}_{1}$ & 2.78 & 2.44 & 2.32 & 2.66 & 2.58 & 2.43 & $1.583^{20}$ & \\
$\mathrm{~T}_{2}$ & 2.90 & 2.59 & 2.48 & 2.71 & 2.63 & 2.51 & $1.568,{ }^{21} 1.594^{20}$ & $1.31^{22}$ \\
$\mathrm{~T}_{3}$ & 2.96 & 2.59 & 2.49 & 2.80 & 2.62 & 2.51 & $1.568,{ }^{21} 1.594^{20}$ & $1.31^{22}$ \\
$\mathrm{~T}_{4}$ & 3.31 & 3.28 & 3.22 & 2.84 & 2.82 & 2.74 & $1.792^{20}$ & \\
$\mathrm{~S}_{1}$ & 0.52 & 0.53 & 0.51 & 0.12 & 0.48 & 0.52 & $0.336^{20}$ & \\
$\mathrm{~S}_{2}$ & 0.54 & 0.53 & 0.52 & 0.14 & 0.48 & 0.52 & $0.336^{20}$ & \\
$\mathrm{~S}_{3}$ & 1.38 & 1.38 & 1.36 & 0.71 & 1.16 & 1.17 & $0.583^{20}$ & \\
\hline
\end{tabular}

\section{S07. Active spaces explored for the silicon vacancy defect}

Here, we compare the $(6,4),(10,10)$ and $(10,12)$ active spaces for the $\mathrm{SiC}_{12}$ impurity cluster in the $\mathrm{SiC}_{126}$ supercell. There is a qualitative agreement between the $(10,10)$ and $(10,12)$ active spaces for both CASSCF and NEVPT2 whereas $(6,4)$ results in a qualitative difference between CASSCF and NEVPT2 state ordering. It is thus necessary to include some orbitals from the conduction band in the active space to account for part of the dynamical correlation. 
Table 14: Singlet and triplet excitation energies (in eV) of $\mathrm{SiV}^{0}$ computed using CAS-DMET and NEVPT2-DMET using the $\mathrm{SiC}_{12}$ impurity cluster shown in Figure $2 \mathrm{~b}$ of the main text. The Table shows excitation energies for the $\mathrm{SiC}_{126}$ using active spaces sizes of $(6,4),(10,10)$ and $(10,12)$.

\begin{tabular}{ccccccc}
\hline \multirow{2}{*}{ State } & \multicolumn{3}{c}{ CAS-DMET } & \multicolumn{3}{c}{ NEVPT2-DMET } \\
\cline { 2 - 7 } & $(6,4)$ & $(10,10)$ & $(10,12)$ & $(6,4)$ & $(10,10)$ & $(10,12)$ \\
\hline $\mathrm{T}_{0}$ & 0.00 & 0.00 & 0.00 & 0.00 & 0.00 & 0.00 \\
$\mathrm{~T}_{1}$ & 2.29 & 2.47 & 2.37 & 2.56 & 2.43 & 2.51 \\
$\mathrm{~T}_{2}$ & 2.42 & 2.60 & 2.51 & 2.51 & 2.45 & 2.55 \\
$\mathrm{~T}_{3}$ & 2.43 & 2.63 & 2.54 & 2.52 & 2.44 & 2.55 \\
$\mathrm{~T}_{4}$ & 3.17 & 3.20 & 3.21 & 2.29 & 2.61 & 2.67 \\
$\mathrm{~S}_{1}$ & 0.70 & 0.58 & 0.52 & 0.35 & 0.42 & 0.48 \\
$\mathrm{~S}_{2}$ & 0.71 & 0.58 & 0.52 & 0.35 & 0.41 & 0.47 \\
$\mathrm{~S}_{3}$ & 1.13 & 1.45 & 1.37 & 1.01 & 0.99 & 1.12 \\
\hline
\end{tabular}

S08. Total energies for the cluster calculations for the silicon vacancy defect

Table 15: Singlet and triplet absolute energies (in a.u.) for the $\mathrm{SiV}^{0}$ computed using CASSCF and NEVPT2 on a finite cluster. All calculations use an active space of $(10,12)$.

\begin{tabular}{ccc}
\hline \multirow{2}{*}{ State } & \multicolumn{2}{c}{ SiVC Cluster } \\
\cline { 2 - 3 } & CASSCF & NEVPT2 \\
\hline $\mathrm{T}_{0}$ & -2377.84613 & -2386.20721 \\
$\mathrm{~T}_{1}$ & -2377.76888 & -2386.13004 \\
$\mathrm{~T}_{2}$ & -2377.76569 & -2386.12785 \\
$\mathrm{~T}_{3}$ & -2377.76348 & -2386.12844 \\
$\mathrm{~T}_{4}$ & -2377.73442 & -2386.12847 \\
$\mathrm{~S}_{1}$ & -2377.82571 & -2386.18753 \\
$\mathrm{~S}_{2}$ & -2377.82565 & -2386.18763 \\
$\mathrm{~S}_{3}$ & -2377.79305 & -2386.16681 \\
\hline
\end{tabular}


S09. Total Energies for supercells considered in the silicon vacancy defect

Table 16: Singlet and triplet absolute energies (in a.u.) of $\mathrm{SiV}^{0}$ computed using CAS-DMET. Here, we use a $(10,12)$ active space for all the systems considered.

\begin{tabular}{ccccccc}
\hline \multirow{2}{*}{ State } & \multicolumn{2}{c}{$\mathrm{SiC}_{52}$} & \multicolumn{2}{c}{$\mathrm{SiC}_{126}$} & \multicolumn{2}{c}{$\mathrm{SiC}_{214}$} \\
\cline { 2 - 7 } & $\mathrm{SiC}_{6}$ & $\mathrm{SiC}_{12}$ & $\mathrm{SiC}_{6}$ & $\mathrm{SiC}_{12}$ & $\mathrm{SiC}_{6}$ & $\mathrm{SiC}_{12}$ \\
\hline $\mathrm{T}_{0}$ & -265.61807 & -265.62052 & -653.62074 & -653.62079 & -1120.62010 & -1120.62045 \\
$\mathrm{~T}_{1}$ & -265.51602 & -265.51620 & -653.53107 & -653.53356 & -1120.53486 & -1120.53721 \\
$\mathrm{~T}_{2}$ & -265.51138 & -265.51145 & -653.52549 & -653.52848 & -1120.52882 & -1120.53088 \\
$\mathrm{~T}_{3}$ & -265.50919 & -265.50918 & -653.52546 & -653.52756 & -1120.52850 & -1120.53067 \\
$\mathrm{~T}_{4}$ & -265.49649 & -265.49521 & -653.50019 & -653.50295 & -1120.50184 & -1120.50430 \\
$\mathrm{~S}_{1}$ & -265.59898 & -265.59901 & -653.60110 & -653.60160 & -1120.60118 & -1120.60211 \\
$\mathrm{~S}_{2}$ & -265.59837 & -265.59848 & -653.60109 & -653.60156 & -1120.60114 & -1120.60200 \\
$\mathrm{~S}_{3}$ & -265.56731 & -265.56728 & -653.56998 & -653.57029 & -1120.57000 & -1120.57041 \\
\hline
\end{tabular}

Table 17: Singlet and triplet absolute energies (in a.u.) of $\mathrm{SiV}^{0}$ computed using NEVPT2DMET. Here, we use a $(10,12)$ active space for all the systems considered.

\begin{tabular}{ccccccc}
\hline \hline \multirow{2}{*}{ State } & \multicolumn{2}{c}{$\mathrm{SiC}_{52}$} & \multicolumn{2}{c}{$\mathrm{SiC}_{126}$} & \multicolumn{2}{c}{$\mathrm{SiC}_{214}$} \\
\cline { 2 - 7 } & $\mathrm{SiC}_{6}$ & $\mathrm{SiC}_{12}$ & $\mathrm{SiC}_{6}$ & $\mathrm{SiC}_{12}$ & $\mathrm{SiC}_{6}$ & $\mathrm{SiC}_{12}$ \\
\hline $\mathrm{T}_{0}$ & -268.18214 & -269.93454 & -654.53519 & -655.17171 & -1121.55130 & -1122.24147 \\
$\mathrm{~T}_{1}$ & -268.08429 & -269.83506 & -654.44040 & -655.07933 & -1121.46190 & -1122.15359 \\
$\mathrm{~T}_{2}$ & -268.08268 & -269.83324 & -654.43865 & -655.07805 & -1121.45889 & -1122.15085 \\
$\mathrm{~T}_{3}$ & -268.07923 & -269.82969 & -654.43876 & -655.07816 & -1121.45923 & -1122.15110 \\
$\mathrm{~T}_{4}$ & -268.07768 & -269.83102 & -653.50019 & -653.50295 & -1121.45069 & -1122.14573 \\
$\mathrm{~S}_{1}$ & -268.17770 & -269.92833 & -654.43151 & -655.07357 & -1121.53213 & -1122.22261 \\
$\mathrm{~S}_{2}$ & -268.17711 & -269.92746 & -654.51758 & -655.15445 & -1121.53216 & -1122.22257 \\
$\mathrm{~S}_{3}$ & -268.15604 & -269.90841 & -654.49254 & -655.13067 & -1121.50827 & -1122.19951 \\
\hline
\end{tabular}


S010. Active spaces explored within this work

(a)

Top view
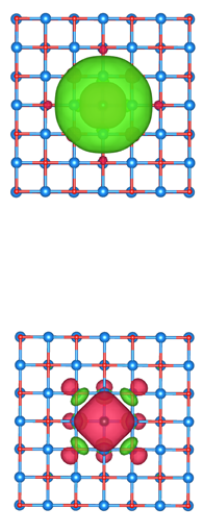

Side view
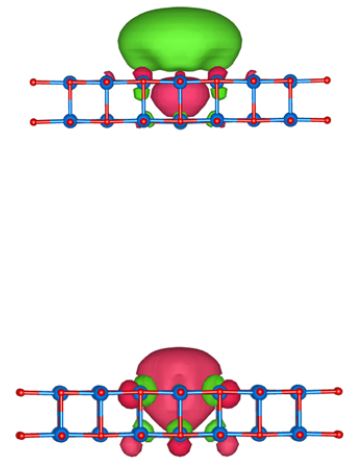

(b)
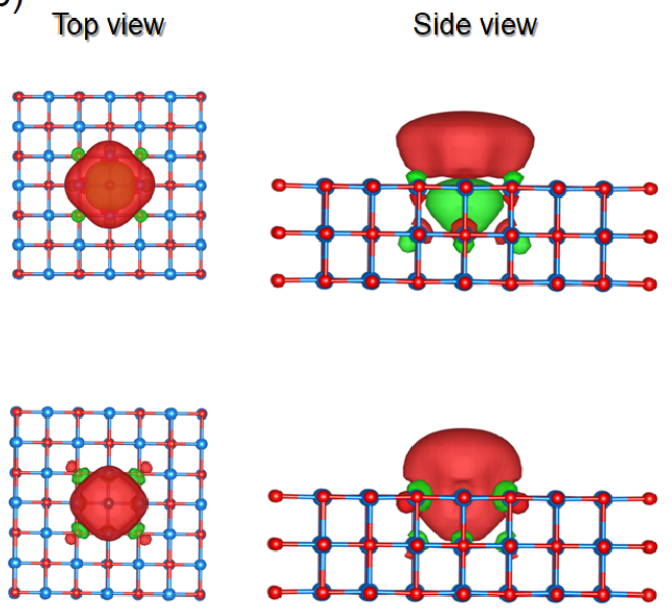

Figure 3: 2,2 active orbitals for (a) double layer $\mathrm{Mg}_{36} \mathrm{O}_{36}$ unit cell and (b) triple layer $\mathrm{Mg}_{54} \mathrm{O}_{54}$ unit cell
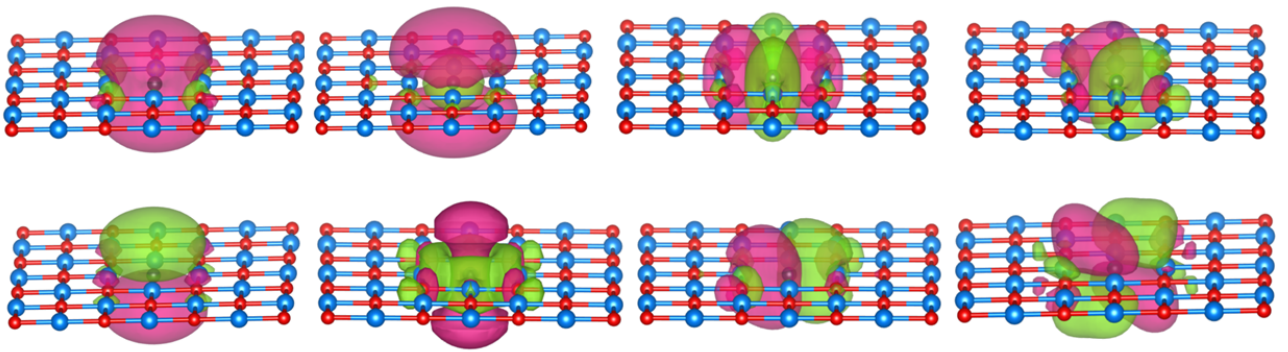

Figure 4: 2,8 active orbitals for single layer $\mathrm{Mg}_{18} \mathrm{O}_{18}$ unit cell
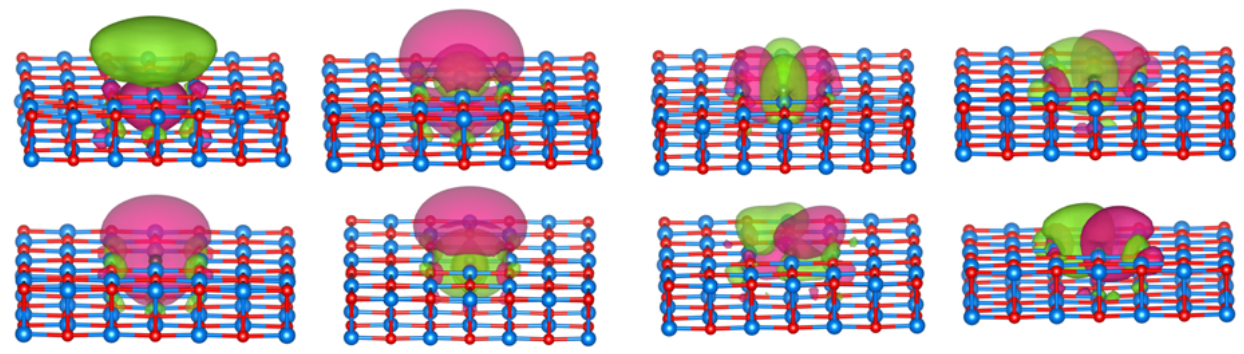

Figure 5: 2,8 active orbitals for double layer $\mathrm{Mg}_{36} \mathrm{O}_{36}$ unit cell 

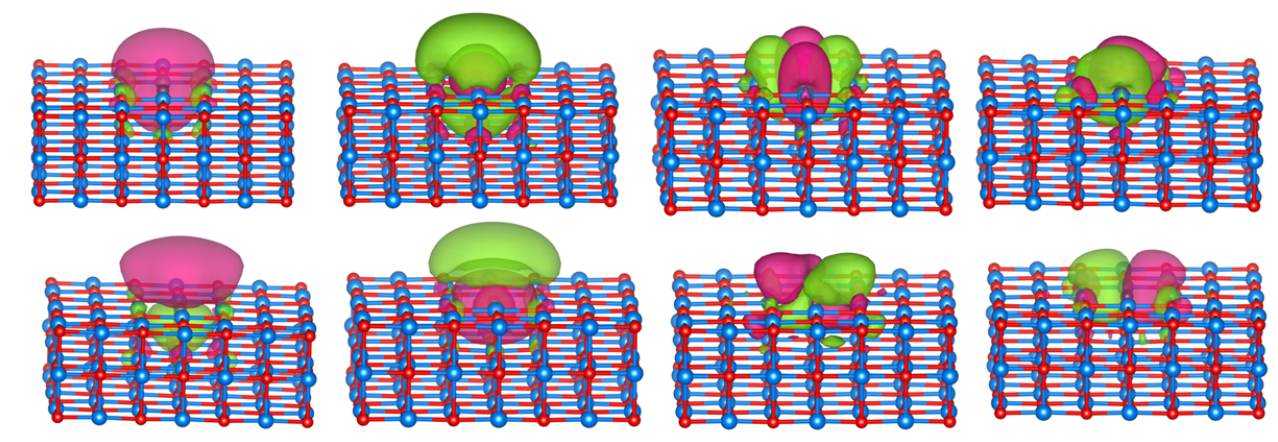

Figure 6: 2,8 active orbitals for triple layer $\mathrm{Mg}_{54} \mathrm{O}_{54}$ unit cell
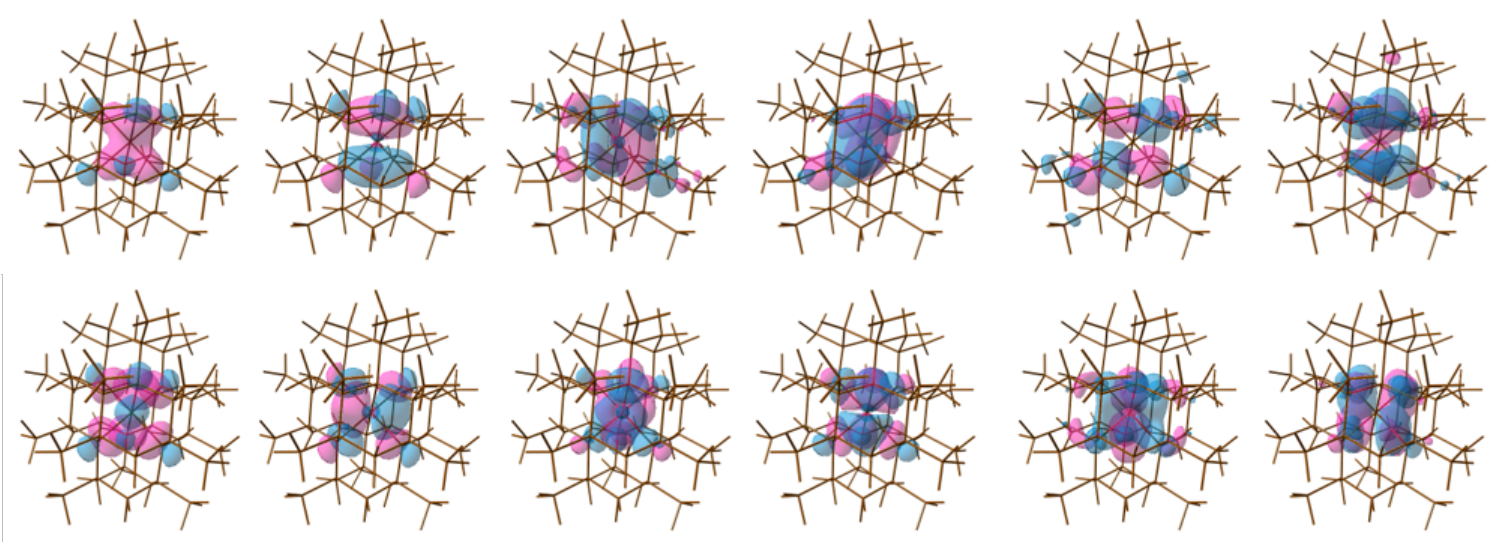

Figure 7: 10,12 active orbitals using the $\mathrm{SiC}_{54} \mathrm{H}_{78}$ finite cluster 

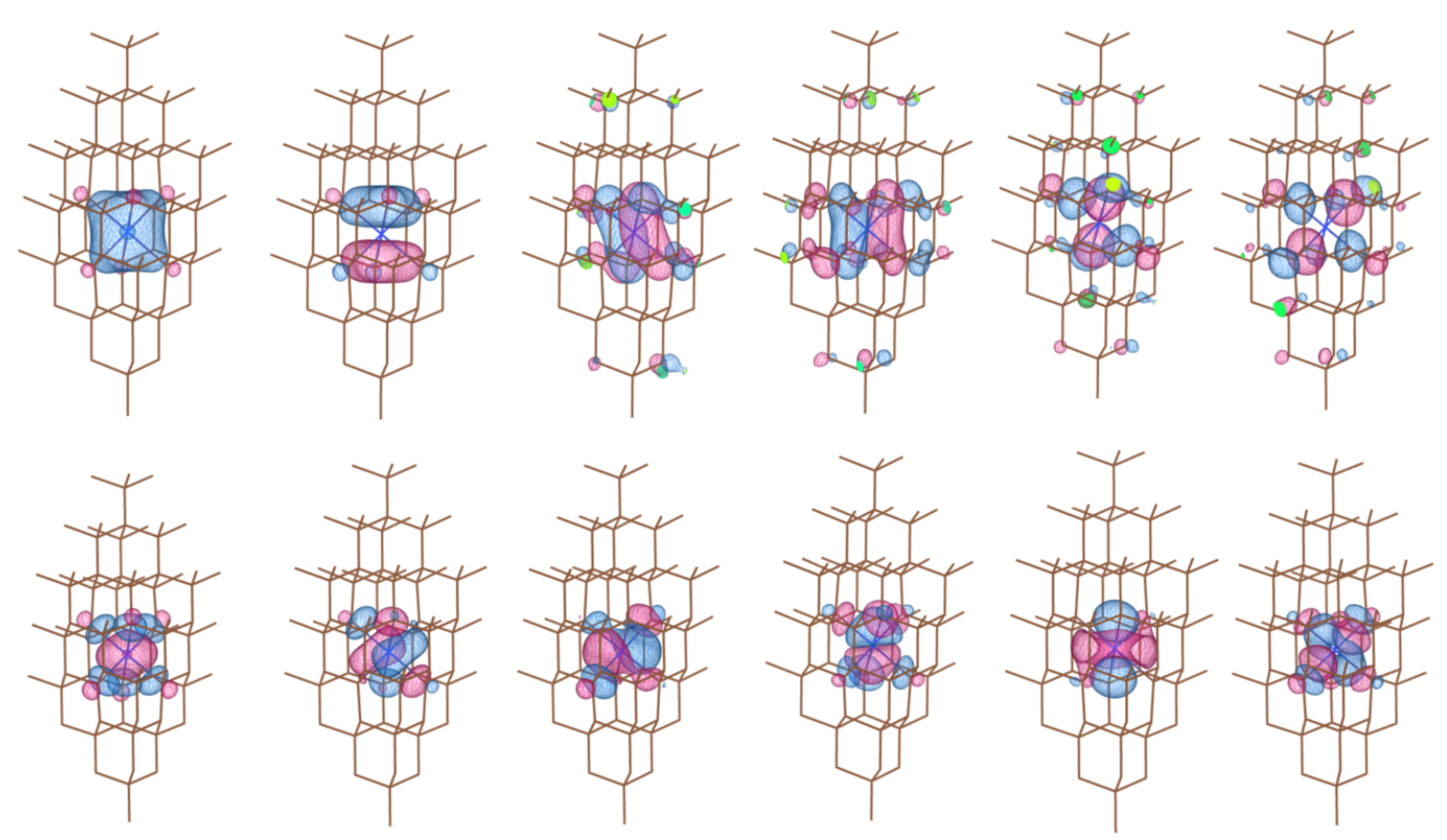

Figure 8: 10,12 active orbitals using the $\mathrm{SiC}_{52}$ unit cell
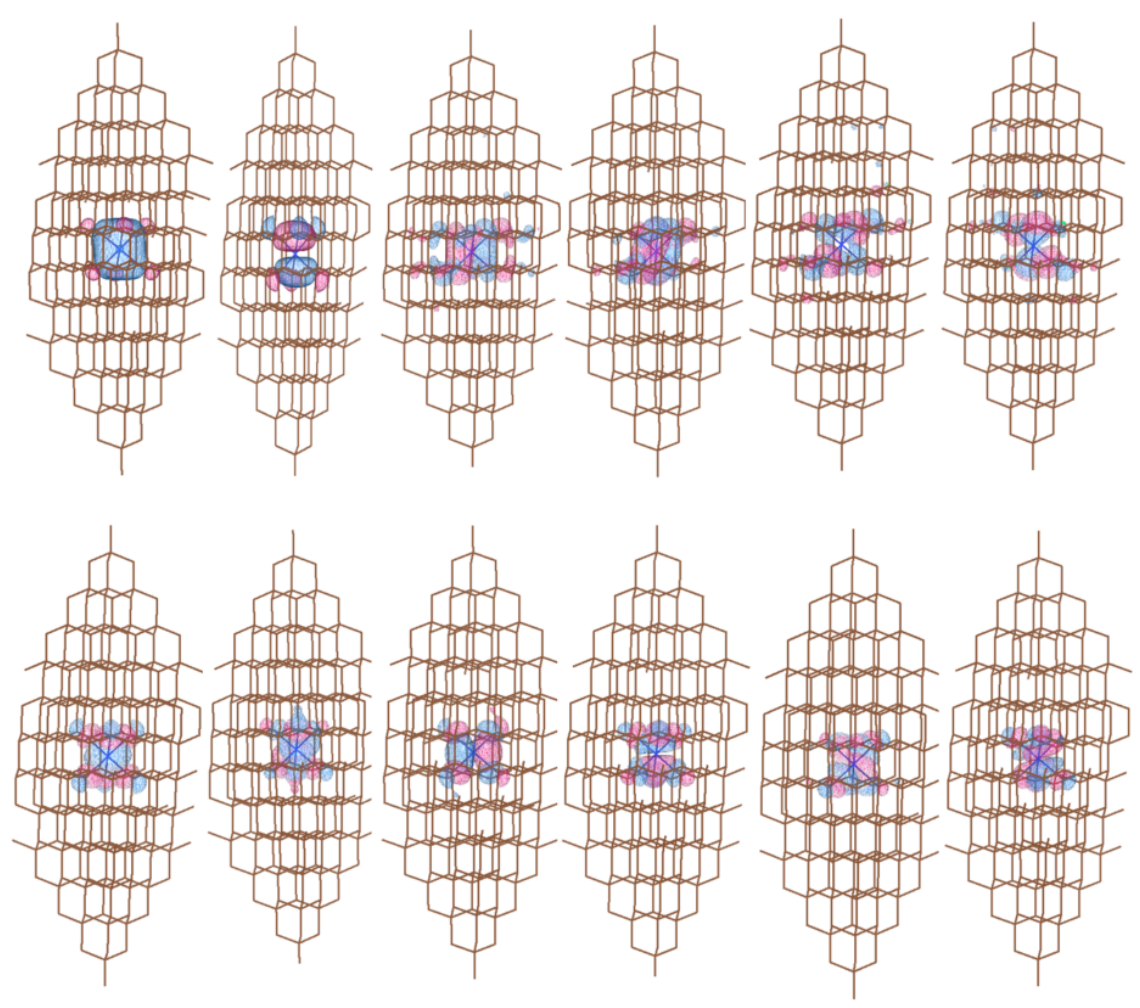

Figure 9: 10,12 active orbitals using the $\mathrm{SiC}_{126}$ unit cell 

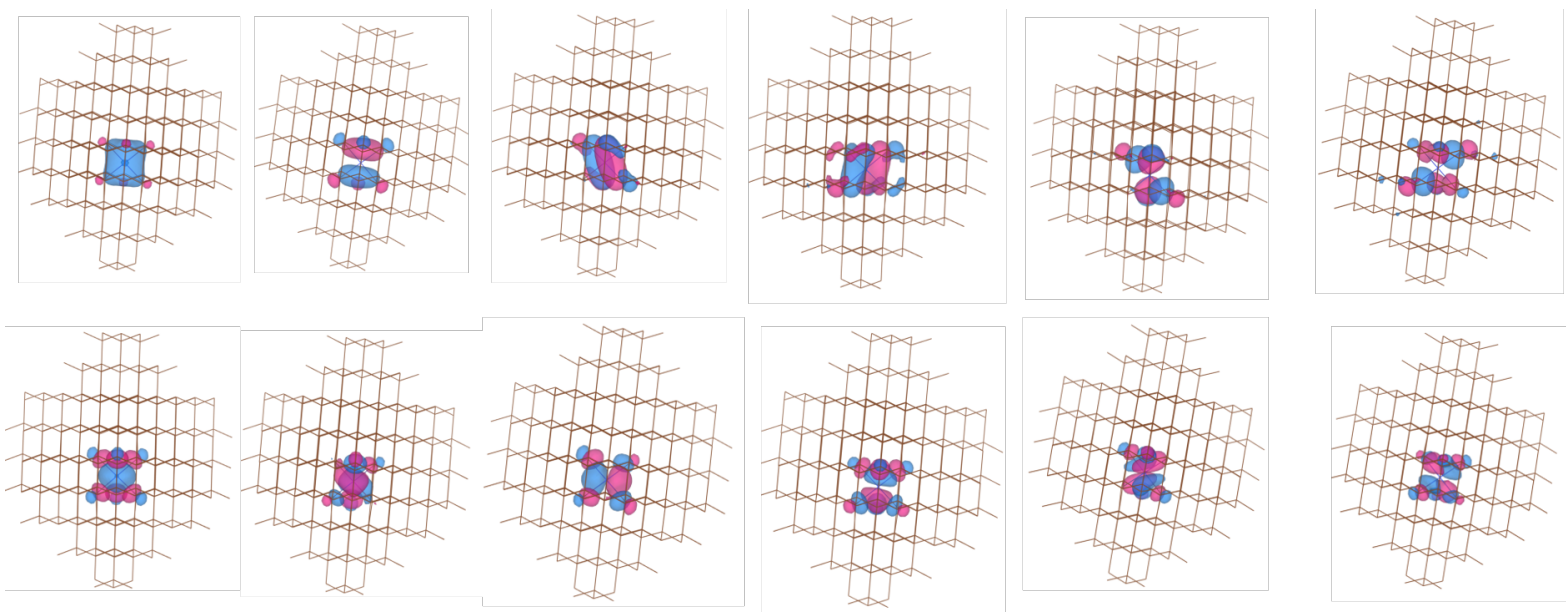

Figure 10: 10,12 active orbitals using the $\mathrm{SiC}_{214}$ unit cell

\section{S011. Natural occupation numbers.}

Table 18: Occupation numbers for the $\mathrm{SiC}_{12}$ impurity cluster used in DMET for the $\mathrm{SiC}_{126}$ unit cell compared to the H-passivated cluster calculations. Orbital numbers correspond to labels used in Figure 11. All the occupation numbers correspond to the $(10,12)$ active space.

\begin{tabular}{|c|c|c|c|c|c|c|c|c|c|c|c|c|c|}
\hline Spin & & \multicolumn{12}{|c|}{ Orbital number } \\
\hline & & 1 & 2 & 3 & 4 & 5 & 6 & 7 & 8 & 9 & 10 & 11 & 12 \\
\hline \multirow{2}{*}{$\mathrm{T}$} & DMET & 1.98 & 1.82 & 1.65 & 1.65 & 1.47 & 1.32 & 0.04 & 0.03 & 0.03 & 0.02 & 0.00 & 0.00 \\
\hline & Cluster & 1.98 & 1.81 & 1.65 & 1.65 & 1.47 & 1.32 & 0.04 & 0.03 & 0.03 & 0.02 & 0.01 & 0.01 \\
\hline & DMET & 1.98 & 1.96 & 1.81 & 1.80 & 1.17 & 1.17 & 0.04 & 0.02 & 0.03 & 0.02 & 0.01 & 0.01 \\
\hline & Cluster & 1.98 & 1.96 & 1.78 & 1.78 & 1.19 & 1.19 & 0.04 & 0.03 & 0.03 & 0.02 & 0.01 & 0.01 \\
\hline
\end{tabular}




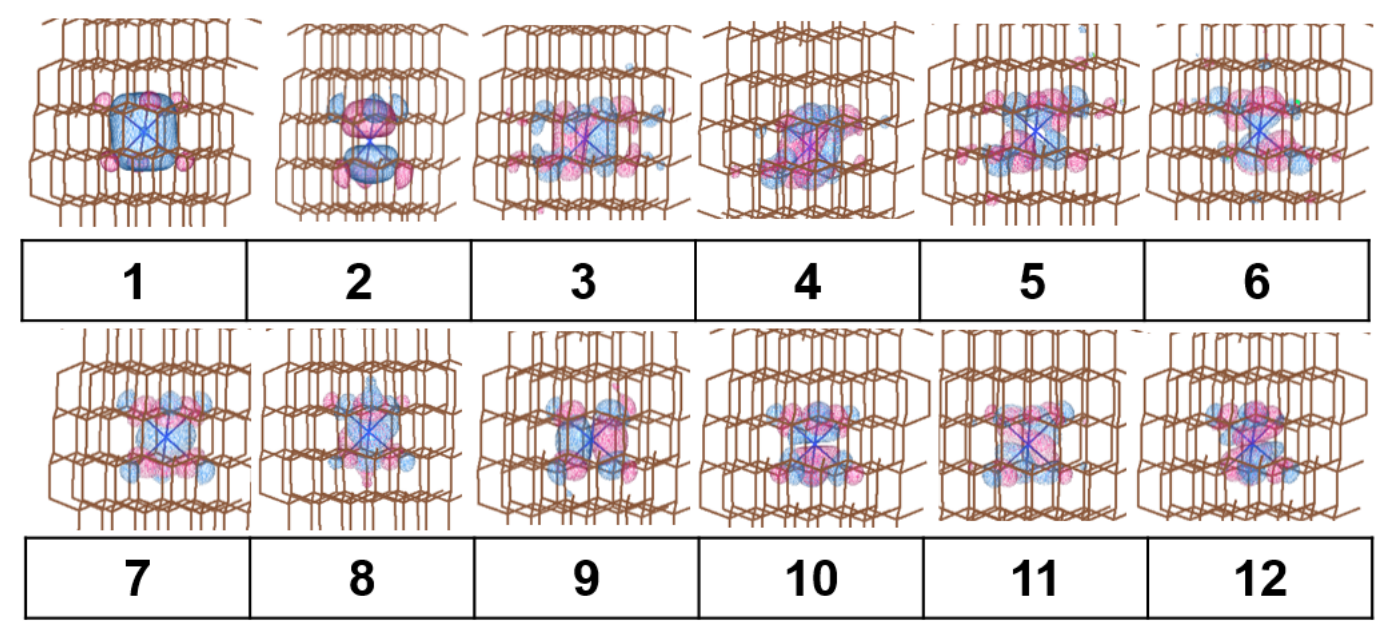

Figure 11: Active orbitals used in the periodic calculations $\left(\mathrm{SiC}_{126}\right)$. The labels below are used to indicate the occupation numbers in these orbitals in tables above. The orbitals similar to the labels for the cluster have been used.

Table 19: Occupation numbers for the $\mathrm{OMg}_{4}$ used in DMET for the $\mathrm{Mg}_{54} \mathrm{O}_{54}$ unit cell compared to the H-passivated cluster calculations. Orbital numbers correspond to labels used in Figure 11. All the occupation numbers correspond to the $(10,12)$ active space.

\begin{tabular}{c|c|c|c|c|c|c|c|c}
\hline Spin & \multicolumn{8}{|c}{ Orbital number } \\
\hline & 1 & 2 & 3 & 4 & 5 & 6 & 7 & 8 \\
\hline $\mathrm{T}$ & 1.00 & 1.00 & 0.00 & 0.00 & 0.00 & 0.00 & 0.00 & 0.00 \\
\hline $\mathrm{S}$ & 1.45 & 1.51 & 0.01 & 0.01 & 0.01 & 0.00 & 0.00 & 0.00 \\
\hline
\end{tabular}

Table 20: Occupation numbers for the $\mathrm{OMg}_{4}$ used in DMET for the $\mathrm{Mg}_{54} \mathrm{O}_{54}$ unit cell compared to the H-passivated cluster calculations. Orbital numbers correspond to labels used in Figure 12. All the occupation numbers correspond to the $(2,2)$ active space.

\begin{tabular}{c|c|c}
\hline Spin & \multicolumn{2}{|c}{ Orbital number } \\
\hline & 1 & 2 \\
\hline $\mathrm{T}$ & 1.00 & 1.00 \\
\hline $\mathrm{S}$ & 1.50 & 0.50 \\
\hline
\end{tabular}




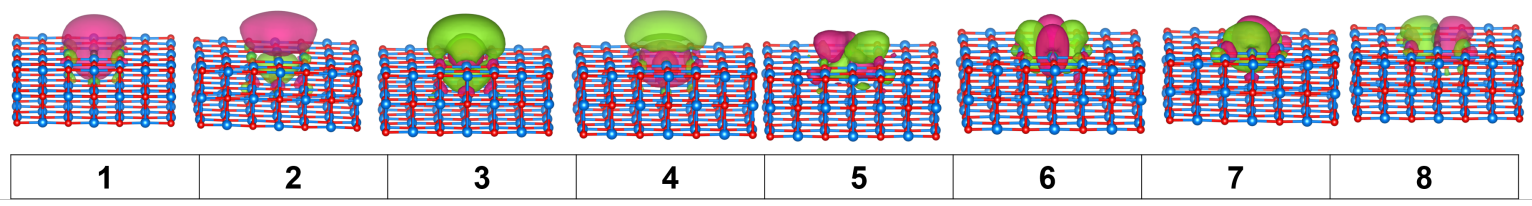

Figure 12: Active orbitals used in $\mathrm{Mg}_{54} \mathrm{O}_{54}$ supercell. The labels below are used to indicate the occupation numbers in these orbitals in tables above. The orbitals similar to the labels for the cluster have been used.

\section{References}

(1) Sun, Q.; Berkelbach, T. C.; Blunt, N. S.; Booth, G. H.; Guo, S.; Li, Z.; Liu, J.; Mc-

Clain, J. D.; Sayfutyarova, E. R.; Sharma, S.; Wouters, S.; Chan, G. K.-L. PySCF: The Python-Based Simulations of Chemistry Framework. Wiley Interdiscip. Rev. Comput. 2018, 8, e1340.

(2) Sun, Q. et al. Recent Developments in The PySCF Program Package. J. Chem. Phys. 2020, 153, 024109 .

(3) Pham, H. Q.; Hermes, M. R.; Gagliardi, L. Periodic Electronic Structure Calculations with The Density Matrix Embedding Theory. J. Chem. Theory Comput. 2020, 16, $130-140$.

(4) Pizzi, G. et al. Wannier90 as A Community Code: New Features and Applications. J. Phys.: Condens. Matter 2020, 32, 165902.

(5) Cui, Z.-H.; Zhu, T.; Chan, G. K.-L. Efficient Implementation of Ab Initio Quantum Embedding in Periodic Systems: Density Matrix Embedding Theory. J. Chem. Theory Comput. 2020, 16, 119-129.

(6) Goedecker, S.; Teter, M.; Hutter, J. Separable Dual-Space Gaussian Pseudopotentials. Phys. Rev. B 1996, 54, 1703-1710. 
(7) Perdew, J. P.; Ruzsinszky, A.; Csonka, G. I.; Vydrov, O. A.; Scuseria, G. E.; Constantin, L. A.; Zhou, X.; Burke, K. Restoring The Density-Gradient Expansion for Exchange in Solids and Surfaces. Phys. Rev. Lett. 2008, 100, 136406.

(8) Kresse, G.; Furthmüller, J. Efficiency of Ab-initio Total Energy Calculations for Metals and Semiconductors Using A Plane-wave Basis Set. Comput. Mater. Sci. 1996, 6, 15 $-50$.

(9) Kresse, G.; Furthmüller, J. Efficient Iterative Schemes for Ab initio Total-energy Calculations using A Plane-wave Basis Set. Phys. Rev. B 1996, 54, 11169-11186.

(10) Kresse, G.; Hafner, J. Ab initio Molecular Dynamics for Liquid Metals. Phys. Rev. B 1993, 47, 558-561.

(11) Kresse, G.; Joubert, D. From Ultrasoft Pseudopotentials to The Projector Augmentedwave Method. Phys. Rev. B 1999, 59, 1758-1775.

(12) Szalay, P. G.; Müller, T.; Gidofalvi, G.; Lischka, H.; Shepard, R. Multiconfiguration Self-Consistent Field and Multireference Configuration Interaction Methods and Applications. Chem. Rev. 2012, 112, 108-181.

(13) Docken, K. K.; Hinze, J. LiH Potential Curves and Wavefunctions for X1sigma+, A1sigma+, B1pi,3sigmat, and 3pi. J. Chem. Phys. 1972, 57, 4928-4936.

(14) Werner, H.; Meyer, W. A Quadratically Convergent MCSCF Method for The Simultaneous Optimization of Several States. J. Chem. Phys. 1981, 74, 5794-5801.

(15) VandeVondele, J.; Krack, M.; Mohamed, F.; Parrinello, M.; Chassaing, T.; Hutter, J. Quickstep: Fast and Accurate Density Functional Calculations using A Mixed Gaussian and Plane Waves Approach. Comput. Phys. Commun 2005, 167, 103 - 128.

(16) Illas, F.; Pacchioni, G. Optical Properties of Surface and Bulk F Centers in MgO from Ab Initio Cluster Model Calculations. J. Chem. Phys. 1998, 108, 7835-7841. 
(17) Henrich, V. E.; Dresselhaus, G.; Zeiger, H. J. Energy-Dependent Electron-Energy-Loss Spectroscopy: Application to the Surface and Bulk Electronic Structure of MgO. Phys. Rev. B 1980, 22, 4764-4775.

(18) Kramer, J.; Ernst, W.; Tegenkamp, C.; Pfnür, H. Mechanism and Kinetics of Color Center formation on Epitaxial Thin Films of MgO. Surf. Sci. 2002, 517, 87-97.

(19) Wu, M.-C.; Truong, C. M.; Goodman, D. W. Electron-Energy-Loss-Spectroscopy Studies of Thermally Generated Defects in Pure and Lithium-Doped $\mathrm{MgO}(100)$ Films on Mo(100). Phys. Rev. B 1992, 46, 12688-12694.

(20) Ma, H.; Govoni, M.; Galli, G. Quantum Simulations of Materials on Near-Term Quantum Computers. Npj Comput. Mater 2020, 6, 85.

(21) Thiering, G.; Gali, A. The $\left(e_{g} \otimes e_{u}\right) \otimes E_{g}$ Product Jahn-Teller Effect in The Neutral Group-IV Vacancy Quantum Bits in Diamond. Npj Comput. Mater 2019, 5, 18.

(22) Green, B. L.; Doherty, M. W.; Nako, E.; Manson, N. B.; D’Haenens-Johansson, U. F. S.; Williams, S. D.; Twitchen, D. J.; Newton, M. E. Electronic Structure of The Neutral Silicon-Vacancy Center in Diamond. Phys. Rev. B 2019, 99, 161112. 